\title{
Generation of rogue waves in gyrotrons with high excess over the threshold
}

\author{
I.V. Zotova ${ }^{1}$, N.S. Ginzburg ${ }^{1}$, M.V. Morozkin, R.M. Rozental ${ }^{1}$, \\ A.S. Sergeev ${ }^{1}$, A.E. Fedotov ${ }^{1}$, V.P. Tarakanov ${ }^{2}$ \\ ${ }^{1}$ Institute of Applied Physics RAS, N.Novgorod, Russia, zotova@appl.sci-nnov.ru \\ ${ }^{2}$ Moscow Engineering Physics Institute, Moscow, Russia
}

Many physical systems exhibit behavior associated with the emergence of the so-called rogue waves which represent dramatically high-amplitude events that occur with low probability but much more frequently than expected in ordinary wave statistics [1]. For example, in laser physics and optics rogue waves arise in the process of supercontinuum generation and represent pulses of ultra broadband light with extremely high peak power [2]. In this paper within the framework of the average approach and in direct 3D PIC (particle-in-cell) simulations we demonstrate that rogue waves can also appear in gyrotrons operating with high excess over the threshold. The mechanism of extreme spikes formation is related to simultaneous cyclotron interaction of a gyrating electron beam with forward and backward waves near the waveguide cutoff frequency as well as with the longitudinal deceleration of electrons. From the practical point of view, experimental observation of the rogue waves generation in gyrotrons can be interesting as a method for production of high-power millimeter-wave radiation with an ultra broadband spectrum. The required exceeding over the threshold can be achieved for routine parameters of the driving electron beams formed by the magnetroninjection guns due to operation at the lowest modes of microwave cavities.

\section{Mechanism of Rogue Waves Generation}

For theoretical analysis of rogue waves generation in gyrotrons we have generalized the self-consistent timedomain model of low-Q gyrotrons [3] in the frame of which the evolution of the field amplitude is described by a non-uniform parabolic equation. In the novel model, first of all an inclination of the beam line with respect to the dispersion characteristic of the waveguide mode is taken into account [4], because the maximum spectrum bandwidth of chaotic generation, including rogue waves, is determined by the difference between resonances with backward and forward waves (points 1 and 2 in Fig. 1 a). The second critical factor which should be included into consideration is the change of the longitudinal momentum of electrons. This effect is not significant for a steady-state generation regime and, as a rule, is ignored in the gyrotron theory. However, in the process of formation of giant ultrashort pulses with a very steep profile strong transverse magnetic fields are initiated even near the cutoff frequency, which, in turn, leads to transformation of the translational energy of electrons to the energy of their orbital rotation and then in the energy of an electromagnetic pulse. Correspondingly, the generalized system of equations has the following form:

$$
\begin{aligned}
& i \frac{\partial^{2} a}{\partial Z^{2}}+\frac{\partial a}{\partial \tau}+\delta(Z) a=i I_{0} J, \\
& \frac{\partial \hat{p}_{\perp}}{\partial Z}+\frac{g_{0}^{2}}{4} \frac{\partial \hat{p}_{\perp}}{\partial \tau}+i \frac{\hat{p}_{\perp}}{\hat{p}_{\|}}\left(\Delta-1+\left|\hat{p}_{\perp}\right|^{2}+\frac{\hat{p}_{\|}^{2}-1}{g_{0}^{2}}\right)=i \frac{a}{\hat{p}_{\|}}, \\
& \frac{\partial \hat{p}_{\|}}{\partial Z}+\frac{g_{0}^{2}}{4} \frac{\partial \hat{p}_{\|}}{\partial \tau}=-g_{0}^{2} \frac{\beta_{\perp 0}^{2}}{2} \operatorname{Re}\left(\frac{\hat{p}_{\perp}{ }^{*}}{\hat{p}_{\|}} \frac{\partial a}{\partial Z}\right) .
\end{aligned}
$$

where the most normalized variables are the same as in the previous gyrotron model [3], $\hat{p}_{\|}=p_{\|} / m v_{\mid 0} \gamma_{0}$ is the normalized longitudinal momentum, $g_{0}=\beta_{\perp 0} / \beta_{\| 0}$ is the initial pitch-factor, the function $\delta(Z)$ describes the profile of a gyrotron resonator. We assume that at the input cross section the electrons are uniformly distributed over cyclotron rotation phases $\hat{p}_{\perp}(Z=0)=e^{i \theta_{0}}, \theta_{0} \in[0,2 \pi)$ and have the same longitudinal momenta $\hat{p}_{\|}(Z=0)=1$. At the system input and output we apply the well-known reflectionless boundary condition [3].

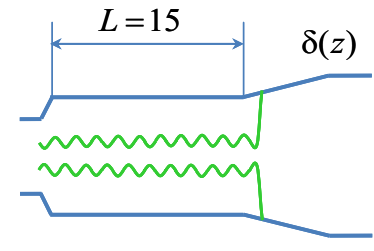

(b)

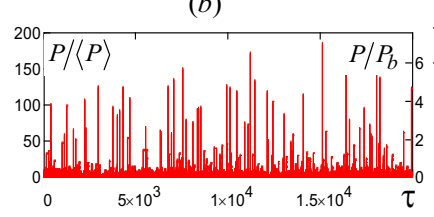

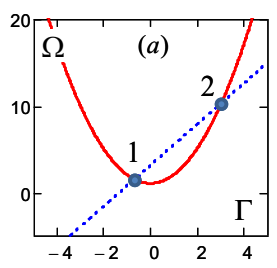

(c)

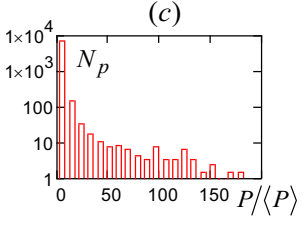

Fig. 1. Gyrotron resonator profile and dispersion diagram $(a)$ of the waveguide mode and the electron beam line for the rogue wave generation $(b)$ with the pulse-height distribution $(c)$.

In Fig. $1 \mathrm{~b}$, c the results of simulations are presented for $L=15, g_{0}=1.3, \beta_{\perp 0}=0.2, \Delta=-0.9$ and $I_{0}=3$. It is seen that radiation represents the sporadic formation of giant pulses with the ratio of the peak power to the average level of $P_{\text {peak }} /\langle P\rangle \sim 150 \div 200$. The corresponding pulse-height histograms have a typical for rogue waves [2] long-tail profile, with the extreme events occurring frequently than expected in the ordinary wave statistics.

The mechanism of giant pulse formation in gyrotrons includes two stages that are illustrated by Fig. 2. At the first stage, electromagnetic radiation is associated with excitation of the backward wave (Fig. 2 a) having a fairly narrow spectrum, corresponding to the low resonance 
point 1 . Upon reflection from the cathode neck, this radiation is partially absorbed by the electron beam, that leads to a dramatic increase in the transverse energy of electrons up to $g \sim 3.5 \div 4$. These electrons produce the forward propagating pulse, which is effectively amplified in the process of its propagation (slippage [5]) along the beam due to the difference between the group velocity of the wave and the translational velocity of particles. This process is accompanied by the pulse front steepening and excitation of a strong transverse magnetic field $H_{\perp} \sim \partial a / \partial Z$. It leads to transformation of the longitudinal to the transverse momenta of particles (Fig. 2 b), that, in turn, provides an additional source of rotational energy for amplification of the forward propagating ultrashort electromagnetic pulse. The appearance of such pulses leads to a significant widening of the output radiation spectrum corresponding to the frequency difference at the resonance points 1 and 2 . It is important that the peak power of generated pulses strongly exceeds not only the mean radiation level but also the kinetic power of the driving electron beam $P_{b}$ (conversion coefficient of $P / P_{b} \approx 6 \div 9$ ).
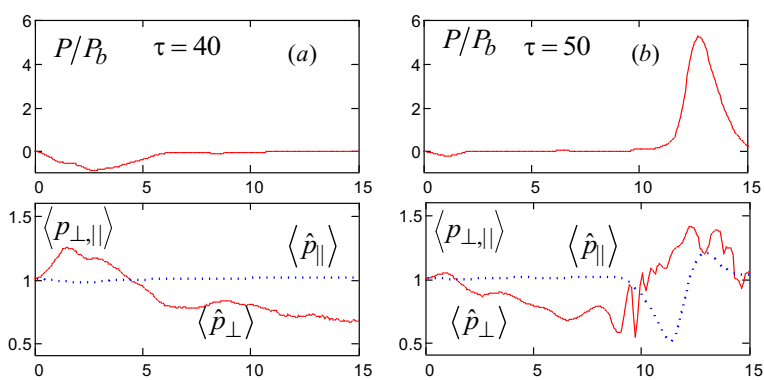

Fig. 2. Two stages of the rogue wave formation in a gyrotron: (a) excitation of the backward wave pumping the transverse energy of later injected particles and $(b)$ deceleration of electrons with transformation of translational energy to the energy of orbital rotation

\section{Results of 3D PIC Simulations}

The possibility of the rogue waves generation in gyrotrons was confirmed by direct 3D KARAT simulations, in which we considered excitation of the $T E_{11}$ mode with the cutoff frequency of $35 \mathrm{GHz}$ by an electron beam with parameters typical for millimeter waveband gyrotrons: particles energy of $20 \mathrm{keV}$, an electron current of $2 \mathrm{~A}$, and a pitch-factor of 1.3. In this case, corresponding normalized parameters are basically the same as those used above (see Fig. 1). Results of the PIC simulations are presented in Fig. 3. It is seen that the gyrotron radiation is characterized by appearance of high-amplitude ultrashort pulses with durations of 0.2 to $0.25 \mathrm{~ns}$ and the peak power of $200 \mathrm{~kW}$, which corresponds to the conversion factor of $\sim 5$. The ratio of the pulse peak power to the mean radiation power reaches 125 , that is in a good agreement with results obtained on the basis of Eqs. (1). It should be noted that strong axial deceleration of electrons was also observed in PIC simulations. From the practical point of view, experimental observation of the rogue waves generation in gyrotrons can be interesting as a method for production of high-power millimeter-wave radiation with an ultrawide spectrum, which, according to our PIC simulations, amounts to $10 \%$ at the level of $10 \mathrm{~dB}$ (Fig. 3 c).

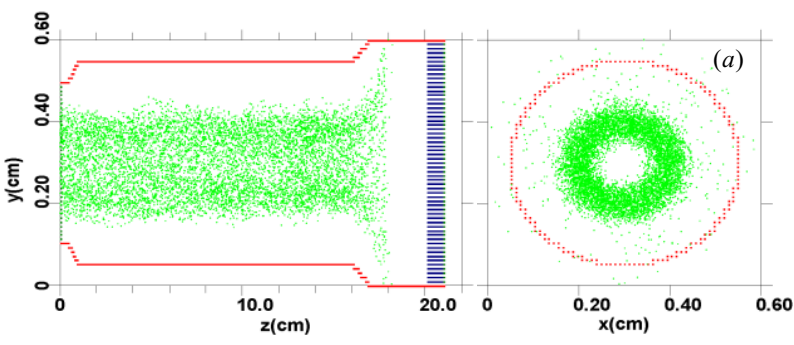

(b)
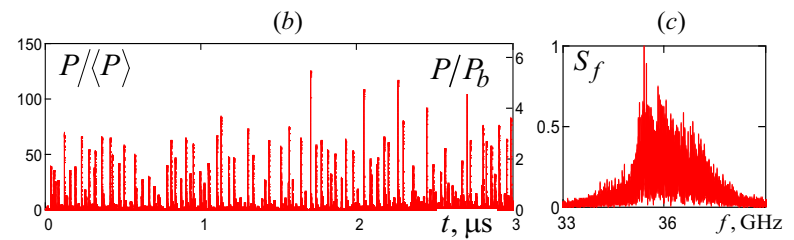

Fig. 3. Results of PIC simulations: (a) geometry of an interaction space, $(b)$ the time dependence of the output power and typical temporal profile of a generated spike; $(c)$ the broadband radiation spectrum

This work was supported by the Russian Foundation for Basic Research (RFBR) (No. 17-08-01077)

\section{References}

1. Onorato, M., Residori, S., Bortolozzo, U., Montina, A., Arecchi, $F$. Rogue waves and their generating mechanisms in different physical contexts // Phys. Reports 2013. V. 528, No. 3. P. 47-89.

2. Solli, D.R., Ropers, C., Koonath, P., Jalali, B. Optical rogue waves // Nature 2007. V. 450, P. 1054-1057

3. Ginzburg, N.S., Nusinovich, G.S., Zavolsky, N.A. Theory of non-stationary processes in gyrotrons with low $\mathrm{Q}$ resonators // Int. J. of Electronics 1986. V. 61, P. 881-894.

4. Ginzburg, N.S., Sergeev, A.S., Zotova, I.V. Time-domain self-consistent theory of frequency-locking regimes in gyrotrons with low-Q resonators // Phys. Plasmas 2015. V. 22, P. 033101.

5. Zhang, T-B., Marshall, T.C. Possibility of generating a high-power self-similar radiation pulse from a free electron laser. // Phys. Rev. Lett. 1995. V.74, Iss.6. P. 916-919. 